\title{
Challenges and the innovations in the care of advanced heart failure patients during COVID-19
}

\author{
Amin Yehya ${ }^{1,2}{ }^{(10} \cdot$ Kevin S. Shah ${ }^{3} \cdot$ Sumeet S. Mitter ${ }^{4} \cdot$ Nasrien E. Ibrahim $^{5,6} \cdot$ Brett Sperry $^{7} \cdot$ Mahek Shah $^{8}$. \\ Sunit-Preet Chaudhry ${ }^{9}$. Navin Rajagopalan ${ }^{10}$. Jaime Hernandez-Montfort ${ }^{11} \cdot$ Rajeev Mohan $^{12}$. \\ Kevin M. Alexander ${ }^{13,14}$. Shashank Sinha ${ }^{15}$. Javed Butler ${ }^{16} \cdot$ Michelle M. Kittleson $^{17}$
}

Accepted: 4 January 2021 / Published online: 12 January 2021

(C) The Author(s), under exclusive licence to Springer Science+Business Media, LLC part of Springer Nature 2021

\begin{abstract}
The COVID-19 pandemic underscored our healthcare system's unpreparedness to manage an unprecedented pandemic. Heart failure (HF) physicians from 14 different academic and private practice centers share their systems' challenges and innovations to care for patients with HF, heart transplantation, and patients on LVAD support during the COVID-19 pandemic. We discuss measures implemented to alleviate the fear in seeking care, ensure continued optimization of guideline directed medical therapy (GDMT), manage the heart transplant waiting list, continue essential outpatient monitoring of anticoagulation in LVAD patients and surveillance testing post-heart transplant, and prevent physician burnout. This collaborative work can build a foundation for better preparation in the face of future challenges.
\end{abstract}

Keywords Heart failure $\cdot$ LVAD $\cdot$ Heart transplantation $\cdot$ COVID-19

\section{Introduction}

Since first reported in Wuhan, China, SARS-CoV2 has infected more than 75.8 million people with an estimated 1.68 million deaths worldwide to date [1]. In the United States (U.S.), the COVID-19 outbreak has caused major disruptions to the healthcare system, highlighting our lack of preparedness to manage a pandemic of this magnitude. In response to these challenges, providers and hospital

Amin Yehya

amin.yehya@yahoo.com

Sentara Advanced Heart Failure Center, Norfolk, VA, USA

2 Eastern Virginia Medical School, Norfolk, VA, USA

3 University of Utah Health Sciences Center, Salt Lake City, UT, USA

4 Icahn School of Medicine At Mount Sinai, Zena and Michael A. Wiener Cardiovascular Institute, New York, NY, USA

5 Massachusetts General Hospital, Boston, MA, USA

6 Harvard Medical School, Boston, MA, USA

7 Saint Luke's Mid America Heart Institute, Kansas City, MO, USA

8 Advanced Heart Failure \& Transplant Center, Jefferson University, Philadelphia, PA, USA systems have been forced to rapidly develop innovations focused on all areas of systems-based healthcare. In this report, physicians from 14 different academic and private practice models across the U.S. (4 West Coast, 8 East Coast, and 2 Midwest) share examples of centers' challenges and innovations highlighting approaches to (1) mitigate fear in seeking medical care, (2) ensure appropriate care delivery and optimization of guideline directed medical therapy, (3) balance risks and benefits in managing care for pre- and

9 Ascension St Vincent Indianapolis, Carmel, IN, USA

10 Newark Beth Israel Medical Center, Newark, NJ, USA

11 Acute Mechanical Circulatory Support Program, Cleveland Clinic Florida, Weston, FL, USA

12 Scripps Clinic Division of Cardiology, La Jolla, CA, USA

13 Division of Cardiovascular Medicine, Stanford Cardiovascular Institute, Stanford, CA, USA

14 Stanford University School of Medicine, Stanford, CA, USA

15 Inova Heart and Vascular Institute, Fairfax, VA, USA

16 University of Mississippi Medical Center, Jackson, MS, USA

17 Smidt Heart Institute, Cedars-Sinai, Los Angeles, CA, USA 
post-transplant patients, (4) pursue surveillance testing in heart transplant and LVAD patients, and (5) protect physicians and staff from burnout, depression, and anxiety. Our work may not be necessarily reflective of all the U.S. centers' experience in caring for patients with advanced heart failure during the COVID-19 pandemic.

\section{Mitigating the fear in seeking medical care}

There is growing evidence that avoiding urgent medical care due to fear of COVID-19 may be as harmful as COVID19 itself. There were over 500,000 more deaths in the U.S. between March and April 2020 compared with the same period over the preceding 6 years, but more than $50 \%$ of those excess deaths were not COVID-19 related [2]. An analysis of 18 medical centers in the U.S. indicated that cardiac catheterization laboratory activations for ST-elevation myocardial infarctions declined by $29 \%$ in March and April 2020 compared with the preceding 14 months [3]. In New York City, out-of-hospital cardiac arrests increased three-fold between March 1 and April 25, 2020, compared with the same period in 2019 [4]. Lack of timely medical care is creating a new category of deaths due to possible delayed care as a result of COVID- 19 .

We must not only convey the risks of COVID-19 to our patients but balance these against the risks of delayed medical care. With the current assiduous measures of screenings and universal masking, the chance of acquiring COVID-19 infection in the hospital is estimated to be $<1 \%$ [5]. And unlike restaurants or shopping malls, where the risk of viral exposure comes without medical benefit, therapy for cardiac conditions tips the scales of the risk-benefit calculus in favor of timely and appropriate medical care. As health care professionals, it is our responsibility to guide our patients to seek necessary care and to influence public perception in favor of proven therapies over excessive fear.

\section{Ensuring appropriate care delivery and optimization of GDMT}

During the COVID-19 pandemic, the rapid uptake and use of telehealth to deliver care have been an unanticipated benefit to many HF and transplant cardiology programs. As payment and regulatory structures have shifted rapidly, the pandemic has provided the opportunity to help our most vulnerable patients to safely communicate virtually with their providers [6]. Salient features of the physical examination can be observed; assessment of lower extremity edema and even jugular venous pressure can be successfully performed over video conference [7]. In fact, telehealth offers the perfect venue for outpatient HF management and
GDMT optimization as the patient's symptoms, vital signs, and laboratory findings can be easily acquired. On the other hand, care delivery through telehealth is a challenge for patients who have no access to $\mathrm{WiFi}$, smartphones, nonEnglish speaking, or are not technologically savvy [8].

The technology of remote monitoring platforms may complement telehealth. Remote monitoring platforms offer the ability to track markers of congestion, identify the need to escalate diuretic or vasodilator therapy, and thus potentially prevent hospitalization. Early data from New York City indicate that effective use of remote pulmonary artery pressure monitoring is associated with a reduction in $\mathrm{HF}$ admissions [9].

\section{Managing patients on the heart transplant waiting list}

Heart transplantation amid a pandemic is fraught with uncertainty (Fig. 1). The precise risk of COVID-19 to transplant patients is unknown, but likely to be higher than the risk in the general population, especially in the early post-transplant period [10]. For patients listed United Network for Organ Sharing status 1 or 2, proceeding with transplantation may outweigh the potential risk of developing COVID-19 in the peri-transplant period. To limit the risk of transmission, most transplant centers require that potential donors and recipients have a negative COVID-19 test at the time of transplant surgery. However, data regarding both the type, timing, and characteristics (i.e., sensitivity, specificity) of testing continue to rapidly evolve.

For those heart transplant candidates on the waiting list with a history of prior COVID-19 infection, it is unclear whether repeat testing is warranted when there is a donor heart offer. Current guidelines suggest that waitlisted patients should be inactivated in the setting of active COVID-19 infection and only reactivated following resolution of symptoms and negative COVID-19 testing at least 14 days following resolution of symptoms [11]. As more data and experience with the virus is obtained, these guidelines will be subject to change.

Ambulatory patients on inotropic therapy or supported with LVAD require a careful discussion of risks, benefits, and alternatives as the risks of waitlist mortality need to be balanced with the risks of peri-transplant COVID-19. Local factors such as disease transmission rate and a given hospital's COVID-19 census will factor into this decision. Shared decision-making with individual patients is of utmost importance. Furthermore, transplant centers will need to ensure that recipients have the resources (both socially and financially) to physically isolate during the peri-transplant period. Patients may need multiple caregivers available to assist in the event that their primary support develops COVID-19 infection. 


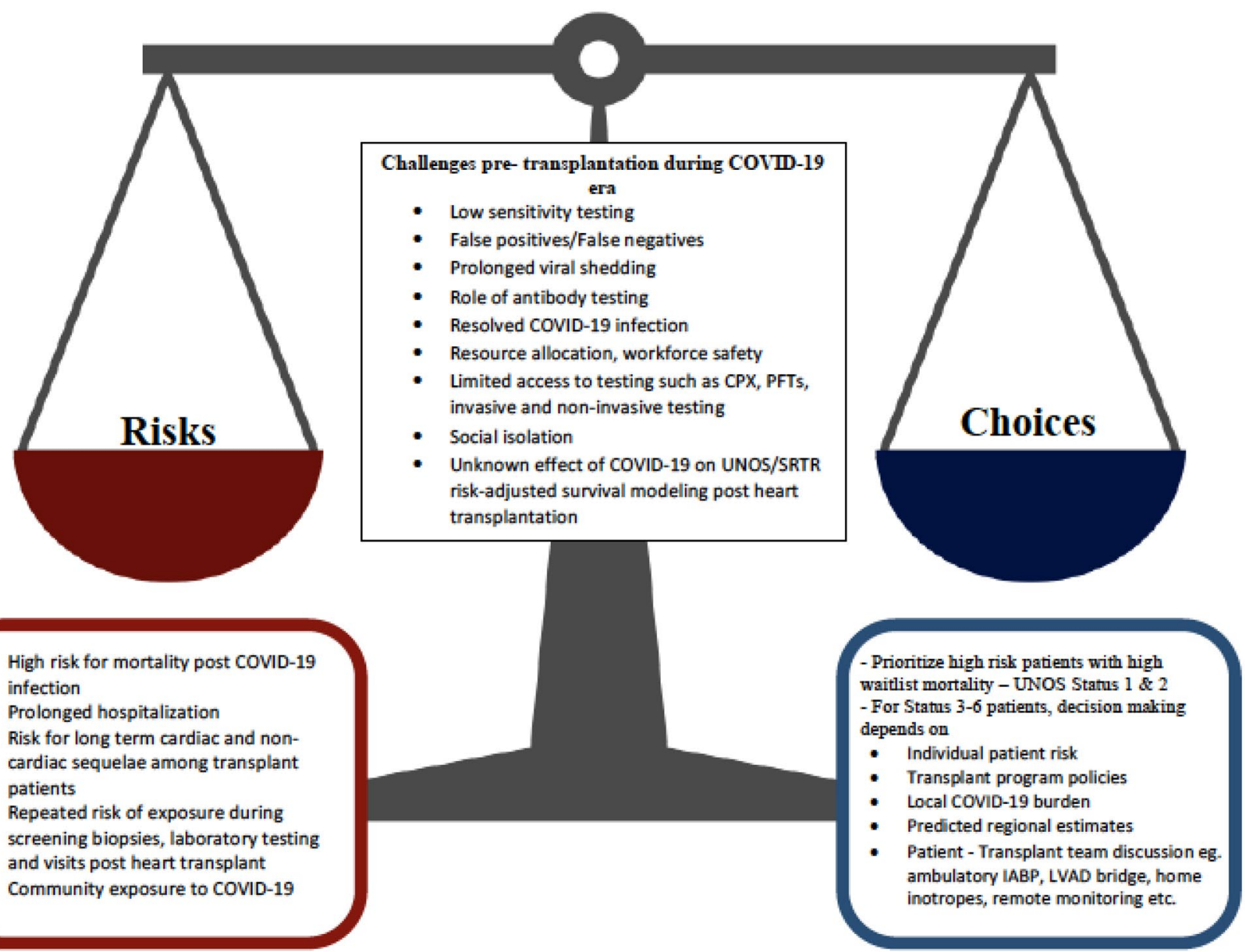

Fig. 1 Challenges in managing patients on the heart transplant waiting list

\section{Surveillance testing and elective procedures in heart transplant and LVAD patients}

Heart transplant patients, particularly those within the first year, require frequent testing and clinical assessments. Using home health services for blood draws can facilitate remote titration of immunosuppression and rejection surveillance (e.g., gene expression profiling and cell-free DNA) instead of endomyocardial biopsies (EMB). These blood-based surveillance tests have been studied in heart transplant recipients $>55$ days post-transplant, although cell-free DNA may potentially may be utilized earlier [12]. The use of these tools can help lower the risk of frequent healthcare system encounters by reducing the number of surveillance biopsies performed.

For outpatients being treated for rejection, when possible, intravenous medications may be switched to oral or subcutaneous therapies (e.g., oral prednisone instead of intravenous methylprednisolone [13]) to limit visits to the healthcare facility. Management of immunosuppression in COVID-19-positive transplant patients requires further study. For those with severe infection (i.e., hospitalized), standard immunosuppression may be temporarily minimized to tacrolimus monotherapy.

Monitoring anticoagulation in LVAD patients is critical to mitigate the risk of device thrombosis and cerebrovascular events. To balance this with the risk of frequent clinic visits, patients with LVADs can be discharged with home INR monitoring machines. For stable outpatients on LVAD support who cannot afford monitoring devices, some centers have partnered with home health agencies and community resources such as the fire department to perform home blood draws.

\section{Ensuring staff and physicians' safety and preventing burnout}

The COVID-19 pandemic has not only magnified preexisting inequities rampant in our healthcare system [14] but it has also magnified the importance of repairing structural 
problems that lead to physician burnout, depression, and anxiety. The challenges physicians face during the pandemic include (1) being deployed in clinically unfamiliar areas, (2) confronting death on a more frequent basis, (3) experiencing the stress of witnessing patients die without loved ones by their sides, and (4) grappling with their own safety and mortality.

Physicians with families have had to simultaneously juggle roles of parents, teachers, and clinicians, all the while ensuring appropriate isolation from loved ones to prevent exposure. COVID-19 has taken a physical and emotional toll on physicians forced to rapidly adapt to a constantly changing and uncertain environment. Many institutions have worked hard to provide support through counseling services, small focus groups for debriefing, and childcare solutions.

The general recognition of health care professions as "healthcare heroes" by hospital administrators, insurers, and the public at large has been a welcome reminder to physicians that their selflessness during the pandemic is valued and that we are measured by more than our efforts in electronic documentation, billing, and relative value units.

Another beneficial consequence of the pandemic has been a renewed focus to de-stigmatize mental health resources to address anxiety and depression among healthcare professionals [15]. Of all the lessons we will carry forward post-pandemic, the essence of why physicians went into medicine should not be lost again.

\section{Conclusion}

Despite the disruptions that COVID-19 had caused to our healthcare systems, there is a silver lining. Our ability to provide care for patients with HF, heart transplantation, and LVADs has quickly adapted to the challenges of the pandemic. The pandemic has led to multiple efforts of global and national collaboration as we work towards navigating the best path for our patients through fear and uncertainty. While this pandemic may end, other crises will manifest, and the lessons learned in response to these challenges will help chart the course for the future.

\section{References}

1. Dong E, Du H, Gardner L (2020) An interactive web-based dashboard to track COVID-19 in real time. Lancet Infect Dis

2. Woolf SH, Chapman DA, Sabo RT, Weinberger DM, Hill L (2020) Excess deaths from COVID-19 and other causes, March-April 2020. JAMA 324(5):510-513

3. Garcia S, Stanberry L, Schmidt C et al (2020) Impact of COVID19 pandemic on STEMI care: an expanded analysis from the United States. Catheter Cardiovasc Interv

4. Lai PH, Lancet EA, Weiden MD et al (2020) Characteristics associated with out-of-hospital cardiac arrests and resuscitations during the novel coronavirus disease 2019 pandemic in New York City. JAMA Cardiol

5. Wong SCY, Kwong RT, Wu TC et al (2020) Risk of nosocomial transmission of coronavirus disease 2019: an experience in a general ward setting in Hong Kong. J Hosp Infect 105(2):119-127

6. Hollander JE, Carr BG (2020) Virtually perfect? Telemedicine for Covid-19. N Engl J Med 382(18):1679-1681

7. Kelly SA, Schesing KB, Thibodeau JT, Ayers CR, Drazner MH (2020) Feasibility of remote video assessment of jugular venous pressure and implications for telehealth. JAMA Cardiol

8. Gorodeski EZ, Goyal P, Cox ZL et al (2020) Virtual visits for care of patients with heart failure in the era of COVID-19: a statement from the Heart Failure Society of America. J Card Fail 26(6):448-456

9. Mitter S, Alvarez-Garcia J, Miller M, Moss N, Lala A (2020) Insights from HeartLogic Multi-sensor Monitoring during the COVID-19 pandemic in New York City. JACC Heart Fail

10. Pereira MR, Mohan S, Cohen DJ et al (2020) COVID-19 in solid organ transplant recipients: Initial report from the US epicenter. Am J Transplant 20(7):1800-1808

11. ISHLT (2020) Guidance from the International Society of Heart and Lung Transplantation regarding the SARS CoV-2 pandemic https://ishlt.org/ishlt/media/documents/SARS-CoV-2_-Guidancefor-Cardiothoracic-Transplant-and-VAD-centers.pdf. Published 2020. Accessed

12. Khush KK, Patel J, Pinney S et al (2019) Noninvasive detection of graft injury after heart transplant using donor-derived cellfree DNA: a prospective multicenter study. Am J Transplant 19(10):2889-2899

13. Kobashigawa JA, Stevenson LW, Moriguchi JD et al (1993) Is intravenous glucocorticoid therapy better than an oral regimen for asymptomatic cardiac rejection? A randomized trial. J Am Coll Cardiol 21(5):1142-1144

14. Abbasi J (2020) Taking a closer look at COVID-19, health inequities, and racism. JAMA 324(5):427-429

15. Taylor WD, Blackford JU (2020) Mental health treatment for front-line clinicians during and after the coronavirus disease 2019 (COVID-19) pandemic: a plea to the medical community. Ann Intern Med

Publisher's Note Springer Nature remains neutral with regard to jurisdictional claims in published maps and institutional affiliations. 